\title{
Advances of Radiation Detector Systems in Nuclear Medicine
}

Eugene Stephane Mananga ${ }^{1,2,3^{*}}$

${ }^{1}$ The Graduate Center, Physics, The City University of New York, 365 Fifth Avenue, New York, New York 10016, USA

${ }^{2}$ Department of Engineering, Physics, and Technology, BCC, The City University of New York 2155 University Avenue, CPH 118, Bronx, New York 10453, USA

${ }^{3}$ Department of Applied Physics, New York University, 6 Metrotech Center, Brooklyn, New York 11201, USA

Corresponding author: Mananga ES, Department of Engineering, Physics, and Technology, BCC, The City University of New York 2155 University Avenue, CPH 118, Bronx, New York 10453, USA; E-mail: emananga1@gc.cuny.edu

Received date: November 22, 2016; Accepted date: November 28, 2016; Published date: November 30, 2016

Copyright: (c) 2016 Mananga ES. This is an open-access article distributed under the terms of the Creative Commons Attribution License, which permits unrestricted use, distribution, and reproduction in any medium, provided the original author and source are credited.

\section{Editorial}

Radiation detector systems are instruments that can identify the presence of radiation in the environment, on the surface and within people. Arguably, no single device can detect all kinds of radiation and no one device is useful in all situations. Understanding of the principles of radiation detection and the characteristics of the commonly encountered detection devices is essential in nuclear medicine imaging. All radiation detector systems share the characteristic that the radiation incident on the transducer produces ionization effects that are not directly observable.

The ideal radiation detector system achieves high quantum detection efficiency by effectively absorbing the radiation of interest. As a US National Institute of Health (NIH-T32) Research Fellow at Massachusetts General Hospital and Harvard Medical School, I had the opportunity to be a member of the Center for Advanced Medical Imaging Sciences now called the Gordon Center for Medical Imaging. During my training, I worked in radiation physics, physics and instrumentation in nuclear medicine [1].

Currently, the Gordon Center for Medical Imaging is developing advances radiation detector systems for nuclear medicine, intraoperative imaging probes, and computed tomography applications to rigorously study and address some of the fundamental obstacles on high-performance imaging systems [2]. Recent works of the Center include but are not limited to the design of novel and costeffective platforms to fabricate radiation detectors using laser processed scintillators [3]. Through the use of laser-induced optical barriers technology, the Gordon Center is investigating new designs of scintillator detectors for PET, SPECT, and CT imaging modalities that were not possible before. The laser-induced optical barriers method consists of focusing a pulsed laser beam inside the scintillator bulk. An optimized pulse with respect to the wavelength, pulse energy, duration, refractive index of the crystal, etc.... can locally alter the crystal structure thus creating an optical barrier that has different index of refraction with respect to its immediate surrounding media [2]. These optical barriers can be used as a surrogate to mechanically pixelate arrays and can bestow high spatial resolution while sustaining the sensitivity in a high-throughput and cost-effective mode.

In early 2016, Sabet et al. [3] published an article that corroborates the feasibility of a new method for fabrication of high spatial resolution CsI:T1 scintillation detectors for SPECT. In 2012, Dr. Majewski [4] gave a remarkable comprehensive lecture at Fermilab on medical applications of radiation detectors. His presentation illustrates how the advances in detector systems can play a major role in the development and future of nuclear medicine, experimental nuclear and high energy physics.
Seco, et al. [5] also published an important article review on the characteristics of radiation detectors for dosimetry and imaging. The article focused on describing the range of technologies used for detecting ionizing radiation in the medical field. It is worth noting that the advances in imaging and therapy will both be driven by advances in detector technology which will pave the way for new types of measurement and improvements in imaging to ensure the safety of staff and patients. Other important recent advances in radiator detector systems are developed in the group of Prof. Levin at Stanford University [6-10]. Levin' group is focused on studying detector systems such as: High-Band Gap Semiconductor Crystal, Cadmium Zinc Telluride [6,7] New Scintillation Light Detection Concepts for PET $[8,9]$ Photo-Detectors for Time of Flight Positron Emission Tomography (ToF-PET) [10].

As in any letter to the editor of this type, many significant and model efforts have not been mentioned. To those whose excellent work has not been mentioned, I have a profound respect for the work that has already been done on the subject matter. I would also like to encourage you to submit any findings to the Journal of Nuclear Medicine and Radiation Therapy. To those who are inspecting new incursion into the field of nuclear medicine and radiation therapy, I invigorate you to join a body of scientists who like to push their intuition and themselves to the boundaries.

Finally, I thank the Editorial office of the Journal of Nuclear Medicine and Radiation Therapy for inviting me to write this editorial article.

\section{References}

1. Mananga ES, El Fakhri G, Schaefferkoetter J, Bonab AA, Ouyang J (2014) Myocardial Defect Detection Using PET-CT: Phantom Studies. PLoS ONE 9: e88200.

2. Gordon Center - MGH - Harvard/research/PET-CT-SPECTInstrumentation.

3. Sabet H, Bläckberg L, Uzun-Ozsahin D, El-Fakhri G (2016) Novel laserprocessed CsI:Tl detector for SPECT. Med Phys 43: 2630.

4. Stan Majewski (2012) Medical Applications of Radiation Detectors.

5. Seco J, Clasie B, Partridge M (2014) Review on the characteristics of radiation detectors for dosimetry and imaging. Phys Med Biol 59: R303R347.

6. Y Gu, Matteson JL, Skelton RT, Deal AC, Stephan EA, et al. (2011) Study of a high-resolution, 3-D positioning cadmium zinc telluride detector for PET Phys Med Biol 56: 1563-1584.

7. Peng P and Levin CS (2010) Design study of a high-resolution breastdedicated PET system built from cadmium zinc telluride detectors. Phys Med Biol 55: 2761-2788. 
Citation: Mananga ES (2016) Advances of Radiation Detector Systems in Nuclear Medicine. J Nucl Med Radiat Ther 7: e117. doi: $10.4172 / 2155-9619.1000 \mathrm{e} 117$

Page 2 of 2

8. Vandenbroucke A, Foudray AM, Olcott PD, C S Levin (2010) Performance characterization of a new high resolution PET scintillation detector. Phys Med Biol 55: 5895-5911.

9. Gu Y, Pratx G, Frances WYL, Levin CS (2010) RADIATION IMAGING PHYSICS Medical Physics 37.
10. Spanoudaki VC, Levin CS (2010) Photo-Detectors for Time of Flight Positron Emission Tomography (ToF-PET) Sensors 10: 10484-10505. 